\title{
Psychosis in Alzheimer's Disease in the National Alzheimer's Disease Coordinating Center Uniform Data Set: Clinical Correlates and Association with Apolipoprotein $\mathbf{E}$
}

\author{
Mary Ann A. DeMichele-Sweet, ${ }^{1}$ Oscar L. Lopez, ${ }^{1,2}$ and Robert A. Sweet ${ }^{1,2,3}$ \\ ${ }^{1}$ Department of Psychiatry, University of Pittsburgh, Pittsburgh, PA 15213-2593, USA \\ ${ }^{2}$ Department of Neurology, University of Pittsburgh, Pittsburgh, PA 15213-2593, USA \\ ${ }^{3}$ VISN 4 Mental Illness Research, Education and Clinical Center (MIRECC), VA Pittsburgh Healthcare System, Pittsburgh, \\ PA 15206, USA \\ Correspondence should be addressed to Robert A. Sweet, sweetra@upmc.edu
}

Received 18 August 2010; Accepted 3 January 2011

Academic Editor: Tatiana Foroud

Copyright ( $) 2011$ Mary Ann A. DeMichele-Sweet et al. This is an open access article distributed under the Creative Commons Attribution License, which permits unrestricted use, distribution, and reproduction in any medium, provided the original work is properly cited.

\begin{abstract}
Approximately 50\% of late-onset Alzheimer's disease (AD) patients develop psychosis $(\mathrm{AD}+\mathrm{P})$, a heritable phenotype associated with more rapid cognitive decline. Prior studies conflict regarding whether apolipoprotein E (APOE) $\epsilon 4$ alleles are associated with $\mathrm{AD}+\mathrm{P}$, possibly due to small sample sizes, inconsistent diagnostic criteria, and different methodologies to assess psychosis. We used the National Alzheimer's Coordinating Center Uniform Data Set to evaluate the largest uniformly characterized sample of AD $+\mathrm{P}$ subjects studied to date for the association of APOE $\epsilon 4$ genotype, along with other demographic and clinical variables. Greater cognitive impairment and depressive symptoms were associated with $\mathrm{AD}+\mathrm{P}$, while the Caucasian race was protective. Neither APOE $\epsilon 4$ carrier status nor allele number was associated with psychosis. The AD+P phenotype is not associated with the APOE $\epsilon 4$ genotype. $\mathrm{AD}+\mathrm{P}$ may represent a useful phenotype for the discovery of non-APOE $\epsilon 4$ genetic variation contributing to the risk of $\mathrm{AD}$.
\end{abstract}

\section{Introduction}

Psychotic symptoms, delusions, and hallucinations are common in Alzheimer's disease $(\mathrm{AD} ; \mathrm{AD}+\mathrm{P})$, occurring in approximately $40 \%$ of individuals over the course of the illness [1]. $\mathrm{AD}+\mathrm{P}$ causes significant distress for patients and family members [2]. $\mathrm{AD}+\mathrm{P}$ is a predictor of worse cognitive and functional outcome, higher likelihood of institutionalization, and higher mortality rate [3]. Importantly, a number of studies indicate that the occurrence of psychosis in $\mathrm{AD}$ is familial [4-6], with an estimated heritability of $61 \%$ [7], indicating a distinct neurobiology of this phenotype [8].

Recently, Ropacki and Jeste [1] comprehensively reviewed the literature on psychosis in $\mathrm{AD}$. They reviewed 55 studies comprised of 9,749 subjects. The most consistent correlate of $\mathrm{AD}+\mathrm{P}$ was greater cognitive impairment than is found in $\mathrm{AD}$ without psychosis $(\mathrm{AD}-\mathrm{P})$. In twenty of thirty studies which assessed this relationship, the prevalence of psychosis increased as cognitive impairment worsened as determined by the Mini Mental State Exam (MMSE). Studies conducted more recently have continued to support the relationship between greater cognitive impairment and $\mathrm{AD}+\mathrm{P}[9,10]$. In contrast, only inconsistent associations have been detected between $\mathrm{AD}+\mathrm{P}$ and age, age at onset of $\mathrm{AD}$, illness duration, gender, and education. $\mathrm{AD}+\mathrm{P}$ may be associated with race, though it has only been examined in a limited number of studies to date [1]. It should be noted that the above conclusions are limited by the inconsistencies across the reviewed studies. Few variables are examined in all studies; sample sizes of individual studies were not always sufficient to detect small-to-moderate effects, and approaches to characterization of subjects, including identification of psychosis, also varied considerably. 
Apolipoprotein E (APOE) is well documented as being an important genetic determinant for the development of lateonset $\mathrm{AD}$ [11]. However, its association with development of psychosis is less clear. We have identified 22 studies that examined the association of the APOE $\epsilon 4$ allele with $\mathrm{AD}+\mathrm{P}$, with nine reporting that $\epsilon 4$ increased the risk for $\mathrm{AD}+\mathrm{P}$, whereas 13 studies found no effect of $\epsilon 4$ [12]. As in the studies of clinical correlates of $\mathrm{AD}+\mathrm{P}$, these reports varied considerably in their subject populations, sample sizes, definitions of $\mathrm{AD}+\mathrm{P}$, and analytic approaches, precluding clear interpretation of the conflicting pattern of results. Moreover, as highlighted by recent genome-wide association studies, genes of small, but real, effects (e.g., RR 1.1-1.2) often show inconsistent results in studies with small sample sizes, with the currently recommended sample sizes numbering in the thousands. Although APOE $\epsilon 4$ confers greater relative risk for $\mathrm{AD}$, it may have a smaller, if any, effect on the development of psychosis in AD.

To more reliably determine the correlates of the $\mathrm{AD}+\mathrm{P}$ syndrome, it would be desirable to analyze a large cohort of individuals with late-onset $\mathrm{AD}$ who have been characterized in a standardized manner. To accomplish this goal we utilized the Uniform Data Set (UDS) collected by the National Alzheimer's Coordinating Center (NACC) to characterize the clinical correlates of $\mathrm{AD}+\mathrm{P}$. To date, this is the largest data set used to look at the association of APOE $\epsilon 4$ with AD+P. We hypothesized that the APOE $\epsilon 4$ genotype is not associated with the development of $\mathrm{AD}+\mathrm{P}$. We also hypothesized that $\mathrm{AD}+\mathrm{P}$ is associated with greater cognitive impairment.

\section{Methods}

2.1. NACC Data Center. The NACC was developed in 1999 with the purpose of developing and maintaining a database that included data from NIH-funded Alzheimer's Disease Centers (ADC) across the country. The UDS was developed by NACC to provide the ADCs with standardized assessments thereby allowing uniformity amongst centers when diagnosing these subjects with mild cognitive impairment or Alzheimer's disease [13]. Individual centers may use additional assessments for their particular research protocols, but every center must complete the UDS assessments [14]. Since 2005, these have included ratings of psychosis on the Neuropsychiatric Inventory Questionnaire (NPI-Q) [15].

2.2. Eligibility Criteria. Subjects were selected for analysis on the basis of having a primary diagnosis of possible or probable AD with an age of onset $\geq 60$. Subjects with comorbid Parkinson's disease or dementia with Lewy bodies were excluded. Additionally, subjects were required to have available psychosis ratings on the NPI-Q and an APOE genotype. Other variables requested, but not available for all subjects, included demographics (age at first visit, age of onset of dementia, sex, race, ethnicity, primary language, education) and scores on the Mini-Mental State Exam (MMSE) [16], global Clinical Dementia Rating Scale (CDR) [17], Hachinski Ischemic Scale [18], and the Geriatric
Depression Scale (GDS) [19]. Because some subjects had multiple visits over time, for these individuals the last available scores for MMSE, CDR (global), Hachinski, and GDS were used for analysis. Regarding race and ethnicity, subjects reported their race to a clinician from the following choices: White, Black, or African-American, AmericanIndian, or Alaska Native, Native Hawaiian or other Pacific Islander, Asian, other, or Unknown. From this, subjects were grouped as Caucasian, African-American, or other for analysis. Subjects also reported as to whether they had Hispanic/ Latino ethnicity. This study had Institutional Review Board approval through the University of Pittsburgh and Universities contributing their data to the NACC.

2.3. Classification of Psychosis. Each subject was assessed for psychosis at each visit using the NPIQ. Informants for the NPIQ ratings were most commonly a subject's spouse (2889, $57.7 \%)$ or child $(1606,32.1 \%)$ and rarely other informants $(515,10.3 \%)$. Subjects were rated positive for psychosis at any visit if they exhibited delusions (question 1) and/or hallucinations (question 2) within a one-month time frame prior to the interview. Subjects with neither item endorsed at any visit were categorized as Never Psychotic. Subjects with one item present at no more than one visit were characterized as having Single Psychosis. Subjects with both items present at any one visit, or one or more items present at multiple visits, were characterized as Multiple/Recurrent Psychosis.

2.4. Statistical Analysis. The association of $\mathrm{AD}+\mathrm{P}$ with baseline clinical and demographic variables was analyzed using univariate (Chi-square and ANOVA, as appropriate) tests. We tested the associations of $\mathrm{AD}+\mathrm{P}$ with $\mathrm{APOE}$ genotype and $\epsilon 4$ carrier status by Chi-square. Follow-up analyses of association used multinomial logistic regression models, including APOE genotype, and clinical and demographic variables. Because MMSE and CDR score are highly correlated, in these latter analyses we omitted the CDR score. Additional multinomial logistic regression analyses were conducted including a main effect of site (24 ADCs contributed to the data set) but were not reported as they yielded essentially identical results.

\section{Results}

We identified 2317 subjects in the NACC database who fulfilled all eligibility criteria for analysis. Of these subjects, $777(33.5 \%)$ had one visit, $730(31.5 \%)$ had two visits, 485 (20.9\%) had three visits, $307(13.2 \%)$ had 4 visits, and 18 $(0.8 \%)$ had five visits for a total number of 5010 visits reported to NACC. The majority of subjects were diagnosed with probable $\mathrm{AD}(2117,91.4 \%)$ and the remaining (200, $8.6 \%$ ) with possible $\mathrm{AD}$. The sample predominately consisted of Caucasian $(1957,84.5 \%)$ and female (1309, 56.5\%) subjects (Table 1). The majority of patients were carriers of the APOE $\epsilon 4$ allele $(1383,59.7 \%)$ (Table 1$)$. 
TABLE 1: Demographic and clinical characteristics and their association with $\mathrm{AD}+\mathrm{P}$.

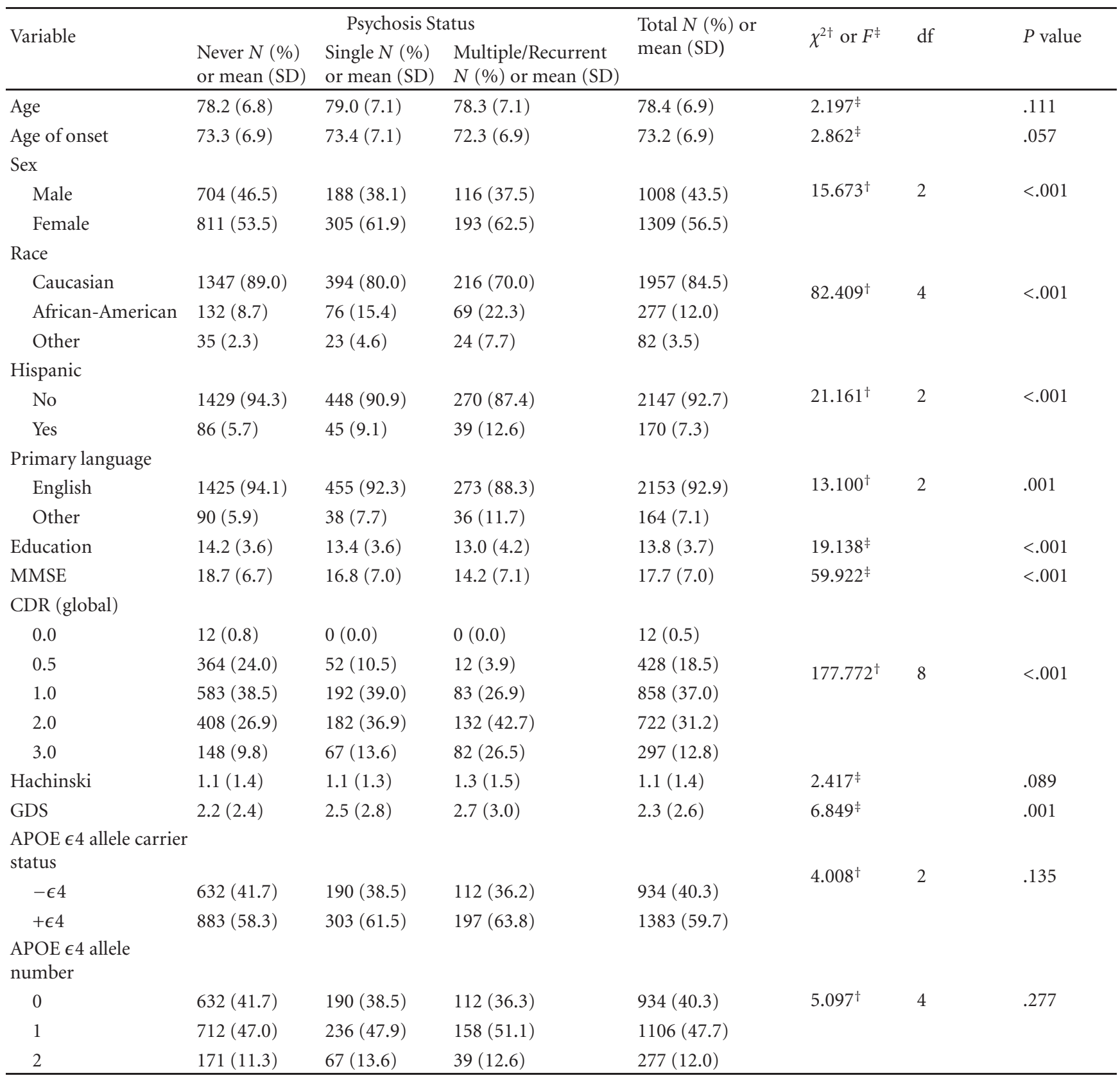

Abbreviations: AD+P, Alzheimer's disease plus psychosis; MMSE, Mini-mental state exam; CDR, Clinical dementia rating scale; GDS, Geriatric depression scale; APOE, Apolipoprotein E.

${ }^{\dagger}$ Pearson's Chi-square test: $\chi^{2}$ values are presented.

${ }^{\ddagger}$ One-way analysis of variance: $F$ values are presented.

Demographic and clinical variables were analyzed for association with $\mathrm{AD}+\mathrm{P}$. Univariate analyses revealed significant associations for psychosis with sex (female), race (nonCaucasian), Hispanic ethnicity, primary language (nonEnglish), lower education, lower MMSE score, higher CDR score, and higher GDS score (Table 1). There were no significant associations of psychosis with age at presentation, age of onset of dementia, Hachinski score, APOE $\epsilon 4$ allele carrier status, or the number of APOE $\epsilon 4$ alleles (Table 1).
Because psychosis does not typically manifest in early stages of $\mathrm{AD}$, classifying someone as a "true $\mathrm{AD}-\mathrm{P}$ " requires individuals to have reached at least mild to moderate stages of disease [6]. Therefore, we conducted follow-up analyses in which individuals classified as $\mathrm{AD}-\mathrm{P}$ were restricted to those who had at least reached a CDR score $\geq 1, N=1941$. In these univariate analyses, significant associations remained for sex, race, Hispanic ethnicity, primary language, education, MMSE, CDR, and GDS. Psychosis was now also significantly 
TABle 2: Demographic and clinical characteristics and their association with $\mathrm{AD}+\mathrm{P}$ with Never Psychotic $(\mathrm{AD}-\mathrm{P})$ cases restricted to $\mathrm{CDR} \geq 1$.

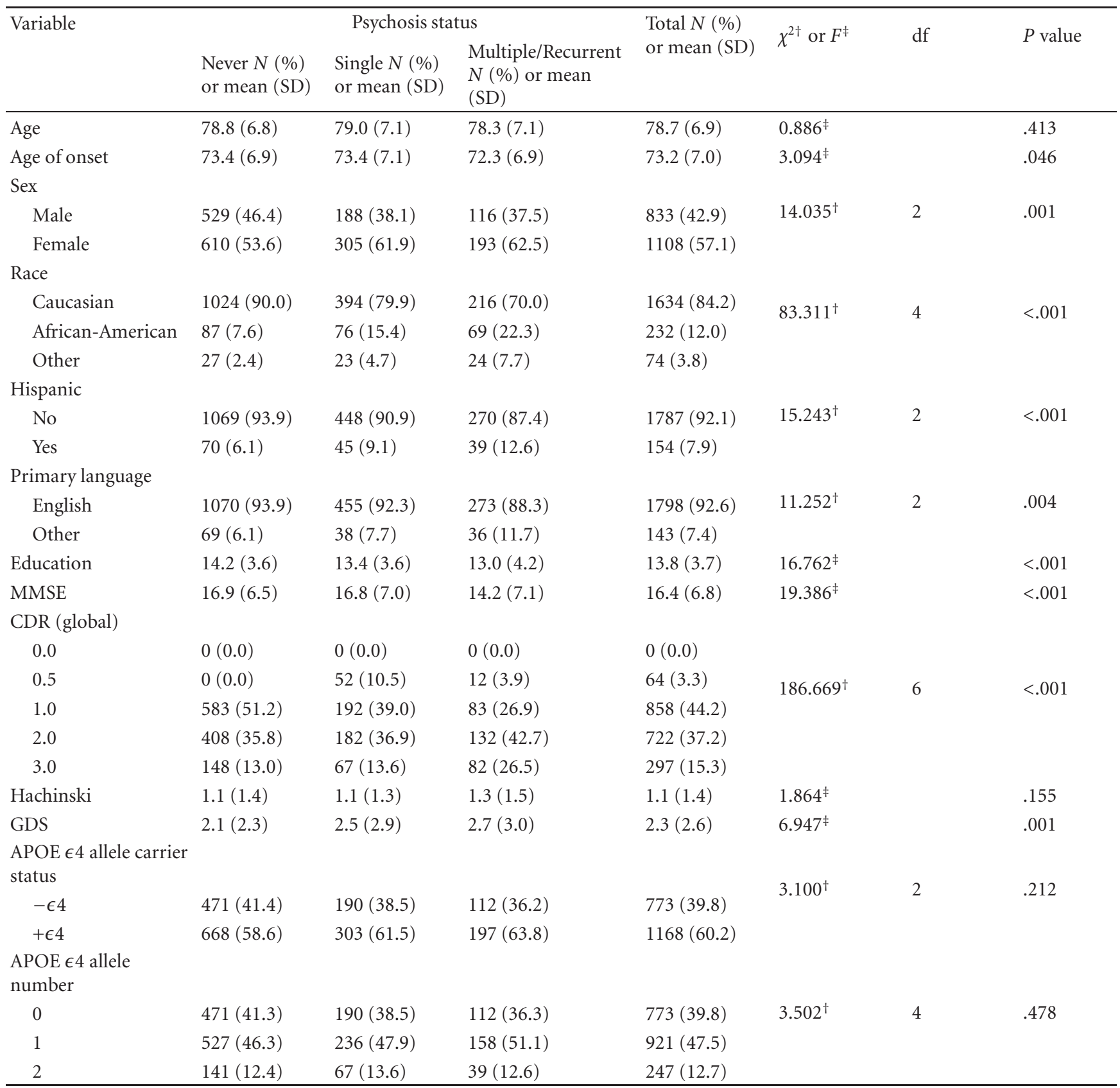

Abbreviations: AD+P, Alzheimer's disease plus psychosis; AD-P, Alzheimer's disease minus psychosis; MMSE, Mini-mental state exam; CDR, Clinical dementia rating scale; GDS, Geriatric depression scale; APOE, Apolipoprotein E.

${ }^{\dagger}$ Pearson's Chi-square test: $\chi^{2}$ values are presented.

${ }^{\ddagger}$ One-way analysis of variance: $F$ values are presented.

associated with age of onset (younger). Age at presentation, Hachinski score, APOE $\epsilon 4$ allele carrier status, and APOE $\epsilon 4$ allele number remained insignificant for association with psychosis (Table 2).

Using multinomial regression, increased age at presentation, lower age of onset of dementia, being non-Caucasian, lower MMSE, and increased GDS were significantly associated with both Single and Multiple/Recurrent Psychosis
(Table 3). Lower education was significantly associated with Single, but not Multiple/Recurrent Psychosis. When AD-P status was restricted to patients with a $\mathrm{CDR} \geq 1$, race and GDS remained significant predictors of both Single and Multiple/Recurrent Psychosis (Table 4). Age, age of onset, and MMSE were significantly associated with only Multiple/Recurrent Psychosis, while education was significantly associated with Single Psychosis. Sex, Hispanic origin, 
TABle 3: Multinomial logistic regression analysis of Single and Multiple/Recurrent Psychosis.

\begin{tabular}{|c|c|c|c|c|c|}
\hline Psychosis category & Variable & OR & $95 \% \mathrm{CI}$ & Wald $\chi^{2}(\mathrm{df}=1)$ & $P$ value \\
\hline \multirow{17}{*}{ Single } & Age & 1.053 & $(1.016,1.092)$ & 8.083 & .004 \\
\hline & Age of onset & 0.960 & $(0.926,0.995)$ & 5.047 & .025 \\
\hline & Sex & 0.799 & $(0.633,1.010)$ & 3.515 & .061 \\
\hline & Race & & & & \\
\hline & Caucasian & 0.457 & $(0.233,0.898)$ & 5.168 & .023 \\
\hline & African-American & 0.834 & $(0.398,1.750)$ & 0.230 & .632 \\
\hline & Other $^{\mathrm{a}}$ & & & & \\
\hline & Hispanic origin & 0.520 & $(0.263,1.029)$ & 3.524 & .061 \\
\hline & Primary language & 1.925 & $(0.901,4.110)$ & 2.862 & .091 \\
\hline & Education & 0.965 & $(0.932,0.999)$ & 4.181 & .041 \\
\hline & MMSE & 0.969 & $(0.951,0.987)$ & 10.924 & .001 \\
\hline & Hachinski & 0.972 & $(0.895,1.056)$ & 0.437 & .508 \\
\hline & GDS & 1.051 & $(1.007,1.096)$ & 5.179 & .023 \\
\hline & APOE $\epsilon 4$ allele number & & & & \\
\hline & 0 & 0.778 & $(0.542,1.115)$ & 1.867 & .172 \\
\hline & 1 & 0.815 & $(0.575,1.154)$ & 1.329 & .249 \\
\hline & $2^{\mathrm{a}}$ & & & & \\
\hline \multirow{17}{*}{ Multiple/Recurrent } & Age & 1.078 & $(1.034,1.123)$ & 12.485 & $<.001$ \\
\hline & Age of onset & 0.914 & $(0.876,0.953)$ & 17.716 & $<.001$ \\
\hline & Sex & 0.847 & $(0.631,1.136)$ & 1.233 & .267 \\
\hline & Race & & & & \\
\hline & Caucasian & 0.311 & $(0.151,0.642)$ & 9.977 & .002 \\
\hline & African-American & 1.008 & $(0.455,2.232)$ & 0.000 & .985 \\
\hline & Other $^{\mathrm{a}}$ & & & & \\
\hline & Hispanic origin & 0.457 & $(0.207,1.007)$ & 3.774 & .052 \\
\hline & Primary language & 1.157 & $(0.495,2.703)$ & 0.113 & .736 \\
\hline & Education & 0.974 & $(0.935,1.014)$ & 1.654 & .198 \\
\hline & MMSE & 0.911 & $(0.892,0.931)$ & 71.322 & $<.001$ \\
\hline & Hachinski & 1.039 & $(0.948,1.140)$ & 0.673 & .412 \\
\hline & GDS & 1.077 & $(1.025,1.132)$ & 8.489 & .004 \\
\hline & APOE $\epsilon 4$ allele number & & & & \\
\hline & 0 & 0.817 & $(0.518,1.290)$ & 0.751 & .386 \\
\hline & 1 & 0.999 & $(0.647,1.541)$ & 0.000 & .996 \\
\hline & $2^{\mathrm{a}}$ & & & & \\
\hline
\end{tabular}

Abbreviations: MMSE, Mini-mental state exam; GDS, Geriatric depression scale; APOE, Apolipoprotein E.

${ }^{\text {a }}$ Reference group.

primary language, Hachinski score, and APOE $\epsilon 4$ allele number were not significantly associated with psychosis in either analysis.

The UDS also requires reporting on several vascular burden and comorbidity variables, such as hypertension. We did univariate analyses of 12 such variables (see Supplementary Table 1 in Supplementary Material available online at doi:10.4061/2011/926597). Only hypertension and diabetes were significantly associated with psychosis. When the analyses were restricted to patients who has at least reached a CDR score $\geq 1$, significance for diabetes remained, but significance for hypertension was lost (Supplementary Table 2). In multinomial regression analyses in which hypertension and diabetes were entered as covariates, along with age, age of onset, sex, race, ethnicity, primary language, education, MMSE, GDS, Hachinski, and APOE, neither was significantly associated with any psychosis measure. The association of all other variables with psychosis remained unchanged (data not shown).

\section{Discussion}

This is the largest study to date to look at the association between APOE $\epsilon 4$ and $\mathrm{AD}+\mathrm{P}$. We were able to examine the associations of APOE and other variables with both any occurrence of psychotic symptoms and with progressively more heritable (and therefore perhaps more biologically relevant) psychotic phenotypes defined by the presence of multiple and/or recurrent psychotic symptoms and with a more stringently restrictive definition of nonpsychotic AD cases. Neither APOE $\epsilon 4$ carrier status nor APOE $\epsilon 4$ allele number was associated with psychosis in any analysis. 
TAble 4: Multinomial logistic regression analysis of Single and Multiple/Recurrent Psychosis when Never Psychotic (AD-P) cases were restricted to $\mathrm{CDR} \geq 1$.

\begin{tabular}{|c|c|c|c|c|c|}
\hline Psychosis category & Variable & OR & $95 \% \mathrm{CI}$ & Wald $\chi^{2}(\mathrm{df}=1)$ & $P$ value \\
\hline \multirow{17}{*}{ Single } & Age & 1.022 & $(0.985,1.061)$ & 1.326 & .250 \\
\hline & Age of onset & 0.980 & $(0.944,1.017)$ & 1.178 & .278 \\
\hline & Sex & 0.833 & $(0.653,1.063)$ & 2.151 & .143 \\
\hline & Race & & & & \\
\hline & Caucasian & 0.482 & $(0.236,0.983)$ & 4.028 & .045 \\
\hline & African-American & 1.046 & $(0.476,2.297)$ & 0.013 & .911 \\
\hline & Other ${ }^{\mathrm{a}}$ & & & & \\
\hline & Hispanic origin & 0.590 & $(0.295,1.178)$ & 2.234 & .135 \\
\hline & Primary language & 1.699 & $(0.787,3.670)$ & 1.822 & .177 \\
\hline & Education & 0.958 & $(0.924,0.993)$ & 5.583 & .018 \\
\hline & MMSE & 1.016 & $(0.995,1.036)$ & 2.221 & .136 \\
\hline & Hachinski & 0.966 & $(0.886,1.053)$ & 0.605 & .437 \\
\hline & GDS & 1.053 & $(1.007,1.101)$ & 5.160 & .023 \\
\hline & APOE $\epsilon 4$ allele number & & & & \\
\hline & 0 & 0.858 & $(0.592,1.243)$ & 0.655 & .418 \\
\hline & 1 & 0.917 & $(0.641,1.313)$ & 0.223 & .637 \\
\hline & $2^{\mathrm{a}}$ & & & & \\
\hline \multirow{17}{*}{ Multiple/Recurrent } & Age & 1.052 & $(1.009,1.098)$ & 5.543 & .019 \\
\hline & Age of onset & 0.929 & $(0.889,0.969)$ & 11.458 & .001 \\
\hline & Sex & 0.876 & $(0.649,1.182)$ & 0.750 & .387 \\
\hline & Race & & & & \\
\hline & Caucasian & 0.326 & $(0.153,0.695)$ & 8.438 & .004 \\
\hline & African-American & 1.204 & $(0.523,2.769)$ & 0.190 & .663 \\
\hline & Other ${ }^{\mathrm{a}}$ & & & & \\
\hline & Hispanic origin & 0.522 & $(0.237,1.151)$ & 2.597 & .107 \\
\hline & Primary language & 1.030 & $(0.440,2.408)$ & 0.005 & .946 \\
\hline & Education & 0.969 & $(0.930,1.010)$ & 2.274 & .132 \\
\hline & MMSE & 0.947 & $(0.925,0.968)$ & 22.376 & $<.001$ \\
\hline & Hachinski & 1.034 & $(0.942,1.136)$ & 0.499 & .480 \\
\hline & GDS & 1.080 & $(1.026,1.137)$ & 8.692 & .003 \\
\hline & APOE $\epsilon 4$ allele number & & & & \\
\hline & 0 & 0.883 & $(0.557,1.398)$ & 0.283 & .595 \\
\hline & 1 & 1.087 & $(0.702,1.685)$ & 0.140 & .709 \\
\hline & $2^{\mathrm{a}}$ & & & & \\
\hline
\end{tabular}

Abbreviations: AD-P, Alzheimer's disease minus psychosis; MMSE, Mini-mental state exam; GDS, Geriatric depression scale; APOE, Apolipoprotein E.

${ }^{\text {a }}$ Reference group.

Similarly, the degree of vascular disease as rated on the Hachinski scale was not associated with $\mathrm{AD}+\mathrm{P}$ in any analysis. Our analysis of these multiple definitions of $\mathrm{AD}+\mathrm{P}$ also revealed other patterns. Greater cognitive impairment and greater depressive symptoms were associated with increased incidence of psychosis across multiple analyses, with the strongest associations observed with Multiple/Recurrent Psychosis. A similar pattern of association was seen for Caucasian race, although it was protective against $\mathrm{AD}+\mathrm{P}$. Other variables demonstrated less consistent associations, emerging (e.g., age and age of onset of $\mathrm{AD}$ ), or disappearing (sex, Hispanic ethnicity, primary language), after controlling for the effects of other variables in multivariate analyses.
Finally, years of education showed associations with $\mathrm{AD}+\mathrm{P}$ in both univariate and multivariate analyses, but not in the latter with the Multiple/Recurrent phenotype.

Many studies have looked at the association of APOE $\epsilon 4$ with $\mathrm{AD}+\mathrm{P}$, with conflicting, albeit largely negative, results [12]. A number of possible reasons might have contributed to these contradictory findings, including the variability in sample size across studies, heterogeneity of $\mathrm{AD}$ subjects (with regard to early versus late stages of $A D$ and early- or late-onset AD), and different methods of psychosis assessment and classification. Adding to the inconsistencies across studies, prior studies varied regarding whether they analyzed APOE genotype, $\epsilon 4$ allele number, or $\epsilon 4$ carrier 
status and the extent to which they included relevant clinical and demographic information in multivariate models.

We were able to overcome many of these problems by utilizing the NACC UDS to evaluate the largest sample of subjects studied for the association of APOE with $\mathrm{AD}+\mathrm{P}$ to date. Subjects from 24 ADCs across the country were assessed using a standardized battery allowing for multivariate analyses including a number of potentially relevant clinical and demographic measures in essentially all subjects. Further, guided by data indicating the stage-dependent emergence of psychotic symptoms during $\operatorname{AD}[6,20]$ and the increased heritability of an $\mathrm{AD}+\mathrm{P}$ phenotype defined by the presence of multiple and/or recurrent symptoms $[6,7]$, we were able to assess the association of APOE with a restrictive definition of $\mathrm{AD}+\mathrm{P}$ likely to be enriched for association with causal genetic variants.

Using these approaches we found no evidence that APOE $\epsilon 4$ is associated with $\mathrm{AD}+\mathrm{P}$. It is possible from the small magnitude of the odds ratios observed in our study that a significant association would be observed in a much larger sample. However, the current results are consistent with the majority of the prior evidence, in which only nine of 22 prior studies, comprising more than 5,200 subjects with $\mathrm{AD}$, found any evidence of significant association [12]. Furthermore, we showed previously that the time from entry into our clinic to the onset of psychosis was not associated with APOE genotypes, lending additional support to the current findings [21]. Thus, the most likely conclusion is that APOE $\epsilon 4$ carrier status and allele number are not associated with psychosis in a population of patients with late-onset Alzheimer's disease.

As with APOE, the current data set and analytic approach can also shed some light on the conflicting results of prior studies which have examined the association of $\mathrm{AD}+\mathrm{P}$ with other clinical and demographic variables. As summarized in a recent review by Ropacki and Jeste [1], $\mathrm{AD}+\mathrm{P}$ was significantly associated with older age in 12/25 studies, with age of onset in 5/12 studies (older in 4/5, younger in $1 / 5$ ), with gender in $7 / 24$ studies (male in $3 / 7$, female in 4/7), with African-American race in 5/7 studies, and with lower education in $4 / 17$ studies. More recent studies have found a significant association between $\mathrm{AD}+\mathrm{P}$ and the severity of depressive symptoms [6, 22-24]. Our findings would suggest that the associations with $\mathrm{AD}+\mathrm{P}$ of age, age of onset of dementia, non-Caucasian race, and depressive symptom severity represent "true positives" while those for sex, ethnicity, and primary language are explained by confounding with other variables.

One of the most consistent correlates of $\mathrm{AD}+\mathrm{P}$ in prior studies is greater burden of cognitive impairment, whether measured as degree of cognitive impairment at time of psychosis or as more rapid cognitive decline [1, 10, 25]. Our current findings are congruent with the former of these prior observations; however, we did not measure the rate of cognitive decline in this study because of the limited number of subjects who have had follow-up assessments entered to date. The mechanisms underlying the association between greater cognitive impairment and $\mathrm{AD}+\mathrm{P}$ are not known, but the current analysis suggests that one potential mediator, increased vascular burden [26], as measured by a global summary, the Hachinski, does not explain the association., We followed this up by analysis of direct measures of vascular risk factors and cerebrovascular disease. Of the 12 UDS variables, only history of hypertension and diabetes achieved marginal significant associations with $\mathrm{AD}+\mathrm{P}$ in univariate analyses, In multivariate analysis the significance for both was lost. Therefore, the most conservative interpretation is that increased vascular burden is not associated with $\mathrm{AD}+\mathrm{P}$.

Amongst the other demographic variables that we tested, the finding that non-Caucasian (predominantly AfricanAmerican) subjects are more likely to develop psychosis during the progression of $\mathrm{AD}$ deserves some comment. It is not clear if this association is due to differences between ethnic groups in allele frequencies of potential $\mathrm{AD}+\mathrm{P}$ risk genes [27] or if being rated as positive for psychotic symptoms, such as persecutory delusions, represents culturally or socioeconomically biased measures of psychosis or referral bias within minority communities. Without having a representative sample of minorities from all socioeconomic backgrounds, it will be impossible to make this distinction. Regardless, the implication for studies of genetic association in $\mathrm{AD}+\mathrm{P}$ is that non-Caucasian subjects should be evaluated separately from Caucasian populations and combined in analyses only if heterogeneity is not observed.

The presence of $\epsilon 4$ alleles of APOE is currently the strongest genetic determinant for late-onset $\mathrm{AD}$, a fact underlined by the findings in most genome-wide association studies of late-onset $\mathrm{AD}$, which have "rediscovered" the association with APOE (http://www.alzgene.org/). We provide evidence that a heritable subtype of $\mathrm{AD}$, defined by the presence of multiple or recurrent psychotic symptoms, is not associated with APOE $\epsilon 4$. As the discovery of non-APOE genetic variation contributing to the risk of late-onset $\mathrm{AD}$ remains a high priority, one approach to enriching cohorts to enhance successful discovery may be to examine the $\mathrm{AD}+\mathrm{P}$ phenotype.

\section{Acknowledgments}

This work was supported by the National Institute on Aging (NIA) Grant U01 AG016976 to the National Alzheimer's Coordinating Center and by AG 027224 and AG 05133. The content is solely the responsibility of the authors and does not necessarily represent the official views of the National Institute of Mental Health or the National Institutes of Health.

\section{References}

[1] S. A. Ropacki and D. V. Jeste, "Epidemiology of and risk factors for psychosis of Alzheimer's disease: a review of 55 studies published from 1990 to 2003," American Journal of Psychiatry, vol. 162, no. 11, pp. 2022-2030, 2005.

[2] D. I. Kaufer, J. L. Cummings, D. Christine et al., "Assessing the impact of neuropsychiatric symptoms in Alzheimer's disease: the neuropsychiatric inventory caregiver distress scale," Journal of the American Geriatrics Society, vol. 46, no. 2, pp. 210$215,1998$. 
[3] N. Scarmeas, J. Brandt, M. Albert et al., "Delusions and hallucinations are associated with worse outcome in Alzheimer disease," Archives of Neurology, vol. 62, no. 10, pp. 1601-1608, 2005.

[4] P. Hollingworth, M. L. Hamshere, P. A. Holmans et al., "Increased familial risk and genomewide significant linkage for Alzheimer's disease with psychosis," American Journal of Medical Genetics Part B, vol. 144, no. 7, pp. 841-848, 2007.

[5] R. A. Sweet, V. L. Nimgaonkar, B. Devlin, O. L. Lopez, and S. T. DeKosky, "Increased familial risk of the psychotic phenotype of Alzheimer disease," Neurology, vol. 58, no. 6, pp. 907-911, 2002.

[6] R. A. Sweet, D. A. Bennett, N. R. Graff-Radford, and R. Mayeux, "Assessment and familial aggregation of psychosis in Alzheimer's disease from the National institute on aging late onset Alzheimer's disease family study," Brain, vol. 133, no. 4, pp. 1155-1162, 2010.

[7] S. A. Bacanu, B. Devlin, K. V. Chowdari, S. T. DeKosky, V. L. Nimgaonkar, and R. A. Sweet, "Heritability of psychosis in Alzheimer disease," American Journal of Geriatric Psychiatry, vol. 13, no. 7, pp. 624-627, 2005.

[8] R. A. Sweet, V. L. Nimgaonkar, B. Devlin, and D. V. Jeste, "Psychotic symptoms in Alzheimer disease: evidence for a distinct phenotype," Molecular Psychiatry, vol. 8, no. 4, pp. 383-392, 2003.

[9] E. A. Weamer, J. E. Emanuel, D. Varon et al., "The relationship of excess cognitive impairment in MCI and early Alzheimer's disease to the subsequent emergence of psychosis," International Psychogeriatrics, vol. 21, no. 1, pp. 78-85, 2009.

[10] P. A. Wilkosz, S. Miyahara, O. L. Lopez, S. T. DeKosky, and R. A. Sweet, "Prediction of psychosis onset in Alzheimer disease: the role of cognitive impairment, depressive symptoms, and further evidence for psychosis subtypes," American Journal of Geriatric Psychiatry, vol. 14, no. 4, pp. 352-360, 2006.

[11] E. H. Corder, A. M. Saunders, W. J. Strittmatter et al., "Gene dose of apolipoprotein E type 4 allele and the risk of Alzheimer's disease in late onset families," Science, vol. 261, no. 5123, pp. 921-923, 1993.

[12] M. A. DeMichele-Sweet and R. A. Sweet, "Genetics of psychosis in Alzheimer's disease: a review," Journal of Alzheimer's Disease, vol. 19, no. 3, pp. 761-780, 2010.

[13] D. L. Beekly, E. M. Ramos, W. W. Lee et al., "The National Alzheimer's Coordinating Center (NACC) database: the uniform data set," Alzheimer Disease and Associated Disorders, vol. 21, no. 3, pp. 249-258, 2007.

[14] J. C. Morris, S. Weintraub, H. C. Chui et al., "The Uniform Data Set (UDS): clinical and cognitive variables and descriptive data from Alzheimer disease centers," Alzheimer Disease and Associated Disorders, vol. 20, no. 4, pp. 210-216, 2006.

[15] D. I. Kaufer, J. L. Cummings, P. Ketchel et al., "Validation of the NPI-Q, a brief clinical form of the Neuropsychiatric Inventory," Journal of Neuropsychiatry and Clinical Neurosciences, vol. 12, no. 2, pp. 233-239, 2000.

[16] M. F. Folstein, S. E. Folstein, and P. R. McHugh, "Mini mental state: a practical method for grading the cognitive state of patients for the clinician," Journal of Psychiatric Research, vol. 12, no. 3, pp. 189-198, 1975.

[17] C. P. Hughes, L. Berg, W. L. Danziger, L. A. Cohen, and R. L. Martin, "A new clinical scale for the staging of dementia," British Journal of Psychiatry, vol. 140, no. 6, pp. 566-572, 1982.

[18] W. G. Rosen, R. D. Terry, P. A. Fuld, R. Katzman, and A. Peck, "Pathological verification of ischemic score in differentiation of dementias," Annals of Neurology, vol. 7, no. 5, pp. 486-488, 1980.
[19] J. A. Yesavage, "Geriatric depression scale," Psychopharmacology Bulletin, vol. 24, no. 4, pp. 709-711, 1988.

[20] W. C. Drevets and E. H. Rubin, "Psychotic symptoms and the longitudinal course of senile dementia of the Alzheimer type," Biological Psychiatry, vol. 25, no. 1, pp. 39-48, 1989.

[21] R. A. Sweet, M. I. Kamboh, S. R. Wisniewski et al., "Apolipoprotein E and alpha-1-antichymotrypsin genotypes do not predict time to psychosis in Alzheimer's disease," Journal of Geriatric Psychiatry and Neurology, vol. 15, no. 1, pp. 24-30, 2002.

[22] M. M. Bassiony, A. Warren, A. Rosenblatt et al., "The relationship between delusions and depression in Alzheimer's disease," International Journal of Geriatric Psychiatry, vol. 17, no. 6, pp. 549-556, 2002.

[23] C. G. Lyketsos, J. M. E. Sheppard, M. Steinberg et al., "Neuropsychiatric disturbance in Alzheimer's disease clusters into three groups: the Cache County Study," International Journal of Geriatric Psychiatry, vol. 16, no. 11, pp. 1043-1053, 2001.

[24] P. A. Wilkosz, C. Kodavali, E. A. Weamer et al., "Prediction of psychosis onset in Alzheimer disease: the role of depression symptom severity and the HTR2A T102C polymorphism," American Journal of Medical Genetics Part B, vol. 144, no. 8, pp. 1054-1062, 2007.

[25] P. A. Wilkosz, H. J. Seltman, B. Devlin et al., "Trajectories of cognitive decline in Alzheimer's disease," International Psychogeriatrics, vol. 22, no. 2, pp. 281-290, 2010.

[26] D. A. Snowdon, L. H. Greiner, J. A. Mortimer, K. P. Riley, P. A. Greiner, and W. R. Markesbery, "Brain infarction and the clinical expression of Alzheimer disease: the Nun Study," Journal of the American Medical Association, vol. 277, no. 10, pp. 813-817, 1997.

[27] R. A. Sweet, B. Devlin, B. G. Pollock et al., "Catechol-Omethyltransferase haplotypes are associated with psychosis in Alzheimer disease," Molecular Psychiatry, vol. 10, no. 11, pp. 1026-1036, 2005. 


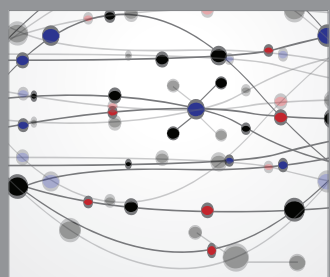

The Scientific World Journal
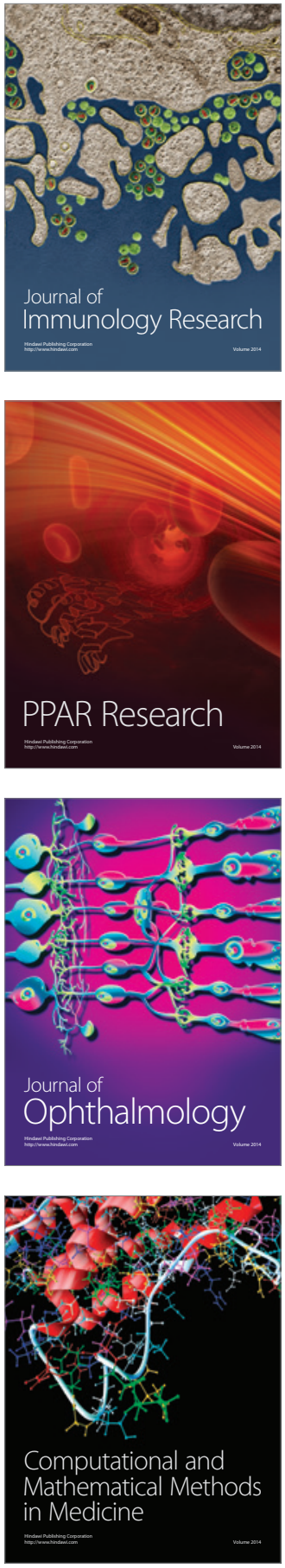

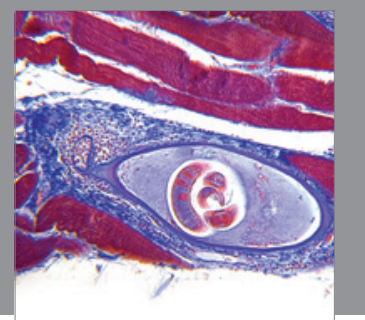

Gastroenterology

Research and Practice
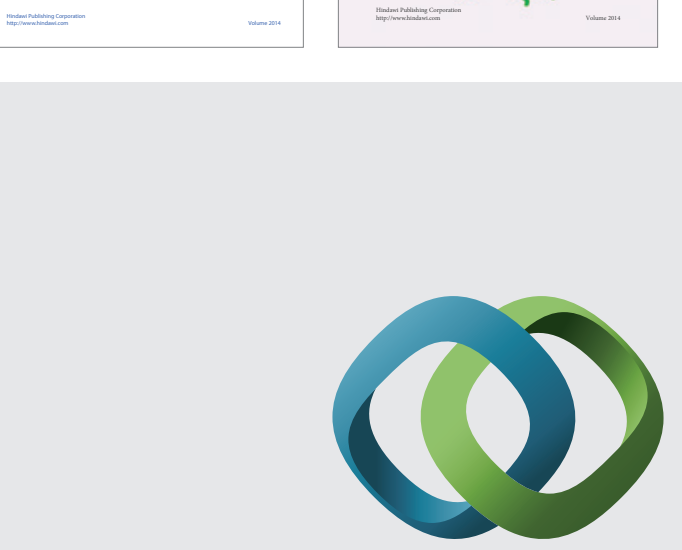

\section{Hindawi}

Submit your manuscripts at

http://www.hindawi.com
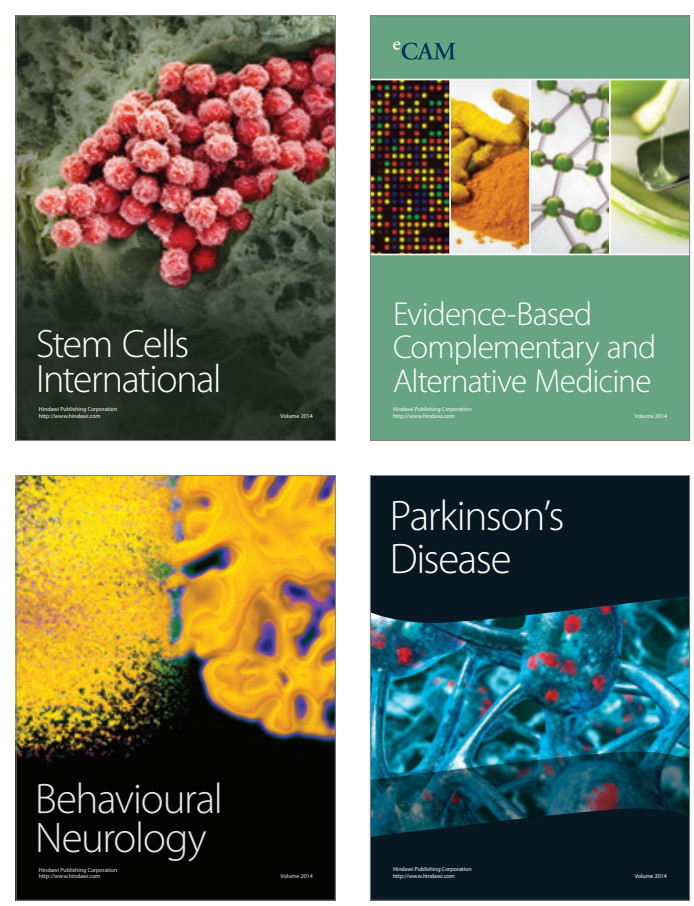

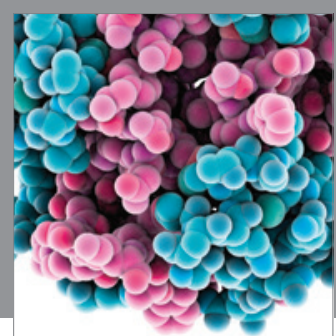

Journal of
Diabetes Research

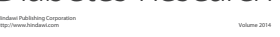

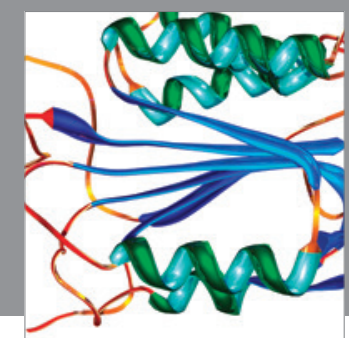

Disease Markers
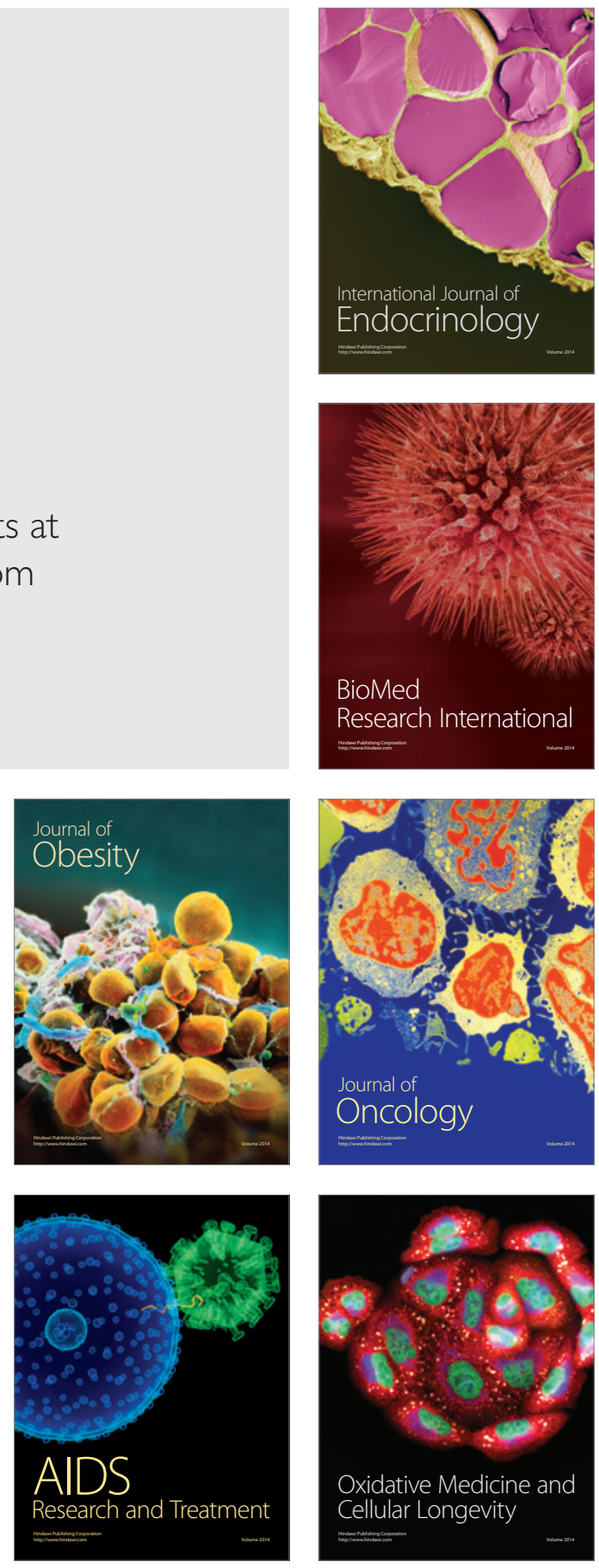